\title{
Angiogenesis subtypes and related differential methylation in renal clear cell carcinoma
}

\author{
Wenzhong Zheng \\ Shiqiang Zhang \\ Huan Guo \\ Xiaobao Chen \\ Zhangcheng Huang \\ Shaoqin Jiang \\ Mengqiang Li
}

\section{Video Byte}

Keywords: tumor angiogenesis, kidney renal clear cell carcinoma (KIRC), microenvironment score, differential methylation sites, master transcription factors, cancer, kidney cancer, epigenetics, multiomics, epigenetic regulation, clear cell renal cell carcinoma, ccRCC, renal cell carcinoma, Cell Communication and Signaling

Posted Date: October 14th, 2021

DOI: https://doi.org/10.21203/rs.3.rs-968283/v1

License: (c) (1) This work is licensed under a Creative Commons Attribution 4.0 International License. Read Full License 


\section{Abstract}

Malignant renal cell carcinoma accounts for approximately $2 \%$ of cancer cases worldwide, and kidney renal clear cell carcinoma (KIRC) is the most common subtype. Tumor angiogenesis is an important prognostic indicator and therapeutic target for KIRC. However, the specific angiogenesis subtypes in KIRC and their epigenetic regulation mechanisms are not well defined. In a recent multi-omics study, researchers analyzed these subtypes in patients using published molecular datasets. Angiogenesis scores calculated based on a 189-gene molecular signature were correlated with several prognostic indicators in over 500 patients. The five genes that contributed most to the scores (MMRN2, CLEC14A, ACVRL1, EFNB2, and TEK) were also associated with overall survival. Clustering analysis based on 183 of the signature genes identified two angiogenesis subtypes among the patients. These subtypes exhibited different angiogenesis scores and prognostic indicator values as well as almost 60,000 differential methylation sites, some of which are related to known tumor angiogenesis-related genes. RFX2, SOX13, and THRA were the main master transcription factors controlling the angiogenic signature. Although further verification is needed, these findings suggest that the 183-gene signature and methylation data can be used to classify the angiogenic subtype of KIRC, which may be useful for the development of targeted treatments. 